Results The implementation of the intervention resulted in a benefit of $€ 3,906,000$ (standard deviation $€ 3,987,000$ ) and 7,200 saved disability-adjusted life years (standard deviation $3,000)$. Including uncertainty and variation for cost elements and performing Monte Carlo simulations (1000 runs) gave insight in the variability in the output. For two stakeholders (employees, society) the output was surrounded with uncertainty but cost-effective. For the third stakeholder (employers), due to the uncertainty, it was not possible to indicate whether the intervention would be cost-effective. The analysis indicated that the most important source for variance was productivity.

Conclusions The described approach gives insight in costs and benefits for different stakeholders in a structured manner. Including a probabilistic approach gives valuable insight in uncertainty and sensitivity of the different cost elements resulting in a more certain outcome of the analysis.

\section{DOES EARLY RETIREMENT REDUCE THE RISK OF MYOCARDIAL INFARCTION? A PROSPECTIVE REGISTRY LINKAGE STUDY OF 617,511 DANISH WORKERS}

${ }^{1} \mathrm{~K} O$ Olesen, ${ }^{2}$ Rugulies, ${ }^{3}$ Hulvej Rod, ${ }^{1}$ Bonde. ${ }^{1}$ Department of Occupational and Environmental Medicine, Bispebjerg Hospital, Copenhagen NV, Denmark; ${ }^{2}$ National Research Centre for the Working Environment, Copenhagen, Denmark; ${ }^{3}$ Section of Social Medicine, Department of Public Health, University of Copenhage, Copenhagen, Denmark

\subsection{6/oemed-2013-101717.402}

Objectives Demographic changes throughout the industrialised world will increase the proportions of retired individuals relative to the active workforce in the coming decades. This will put a substantial financial strain on the economy. Recent studies suggested that early retirement may have beneficial effects on health outcomes. In this study we examined if the risk of myocardial infarction (MI) was reduced following retirement in a Danish population sample.

Methods Participants were 617,511 Danish workers, born between 1932 and 1948 entering the study at the age of 60, without previous known incidents of MI or ischemic heart disease. Information on retirement and MI were obtained from Danish national registers. The participants were followed-up from the week they turned 60 years until event (incident MI) or censuring due to death to causes other than MI, migration, absence from the labour market of more than 26 weeks, or end of 8 years of follow up, whichever came first. We used Cox proportional hazard model to address the relation between retirement and onset of MI, while adjusting for age, sex, income, jobgroup, education, cohabitation and ethnicity.

Results Three per cent of the population was diagnosed with MI during follow up. Retirement was associated with a modestly increased risk of $\mathrm{MI}(\mathrm{OR}=1.11 ; 95 \% \mathrm{CI}=1.06$ to 1.16$)$ when comparing retired workers with active workers of the same age. Further analyses stratified by covariates yielded similar results.

Conclusions This study does not support the hypothesis that early retirement reduces risk of MI. On the contrary, we found that retirement was associated with a modestly increased risk of MI.

\section{OCCUPATIONAL SAFETY AND HEALTH IMPACT ASSESSMENT; HEALTH, SOCIO-ECONOMIC AND COST EFFECTS OF EXCLUDING SMALL LOW RISK COMPANIES OF THE RIE-OBLIGATION}

S G van den Heuvel, Geuskens, Vergeer, Treutlein, de Weerd, Dhondt. TNO, Hoofddorp, Nederland

\subsection{6/oemed-2013-101717.403}

Objectives Policy makers have an urgent need for quantitative data to support their decision-making process. More and more quantitative data are available, but the main problem remains how to construct useful information from all this data. The TNO Occupational Safety and Health Impact Assessment (OSHIA) ex ante tool helps policy makers with ex ante impact assessment in the field of occupational safety and health. This paper applies this method on a case example, the abolishment of the obligation to carry out a RIE for companies (1-9 employees) in low risk sectors in the Netherlands.

Method A stepwise quantitative ex ante evaluation was carried out following the TNO OSHIA framework. To gain insight into the affected population, compliance with the current legislation, and the effect of (abolishment of) a RIE on work-related exposures, health (MSD, depressive symptoms), QALY's, and socioeconomic effects (sick leave, productivity). Data were used from the Netherlands Working Conditions Survey (NWCS), the Employers' Labour Survey (WEA), and the literature (QALY, productivity). A cost-benefit analysis was carried out to estimate the change in costs for employees, employers and society if the RIE would be abolished. The analysis was based on several scenarios of the change in exposure. Within each scenario a sensitivity analysis was included.

Results The examined change in legislation would affect approximately 250,000 employees in approximately 65,000 companies. Of these companies, only $27 \%$ carried out a RIE in 2010 . In most scenarios the health and socio-economic effects were small. Conclusions The stepwise approach of the ex ante impact assessment proved to be useful to estimate the consequences of a policy change in OSH. In particular, the use of scenarios and the analysis of the uncertainty provides insight that facilitates policy decisions.

\section{LONGITUDINAL ASSOCIATIONS BETWEEN FLIGHT SCHEDULES AND OCCUPATIONAL ACCIDENTS AMONG CABIN CREW}

${ }^{1}$ A van Drongelen, ${ }^{1}$ Boot, ${ }^{1}$ Pas, ${ }^{2}$ Penders, ${ }^{3}$ Hlobil, ${ }^{1}$ van der Beek, ${ }^{1}$ Smid. ${ }^{1}$ VU University Medical Center/EMGO Institute for Health and Care Research, Amsterdam, Nederland; ${ }^{2}$ Health-Works, Naarden, Nederland; ${ }^{3}$ KLM Health Services, Schiphol Airport, Nederland

\subsection{6/oemed-2013-101717.404}

Objectives Work schedules of cabin crew involve early starts, long working hours, night flights and the crossing of time zones. This can impose disturbed sleep, fatigue, lack of alertness, and possibly cause occupational accidents onboard. Although it has been shown that the risk for occupational accidents increases over successive shifts, it is unclear what kind of longitudinal flight schedule exposure affects their incidence. Therefore the objective of this study is to examine the associations between cumulative exposure to different flight schedules and the occurrence of occupational accidents among cabin crew.

Methods Data from the five-year historic MORE cohort was used. The study population consisted of 6311 cabin crew members from this cohort. For each employee, daily flight schedules from 2005 until 2008, and registered occupational accidents in 2009 were collected. The association between the cumulative exposure to different types of flight schedules and the 
occurrence of occupational accidents was determined using multivariate logistic regression analyses. Multivariate analyses were adjusted for age, gender, marital status, children (yes/no), job title, multiple employers (yes/no) and the number of flights flown during 2005-2008.

Results In 2009, 289 accidents were reported. Multivariate analyses showed that the number of short-haul flights during 20052008 was positively associated with the occurrence of occupational accidents in $2009(\beta=0.004 ; p=0.012)$. Cumulative exposure to long-haul flights during 2005-2008 proved to be negatively associated with occupational accidents in 2009 ( $\beta=$ $-0.006 ; p=0.011$ ).

Conclusions The results of this study show that cumulative exposure to short-haul flights is associated with an increased risk for occupational accidents among cabin crew. This increased risk may be caused by the specific characteristics of short-haul flights, such as frequent customer service and a high time pressure. Future research should focus on the effects of alternation between different flight schedules and the influence of specific roster characteristics of short-haul schedules.

\section{EFFECTS OF EXPOSURE REDUCTION ON OCCUPATIONAL ALLERGIC DERMATITIS: COMPARISON OF PREVENTION EFFECTIVENESS OF NATIONAL ACTIONS IN UK AND FRANCE}

${ }^{1}$ L B C Bensefa-Colas, ${ }^{2}$ Telle-Lamberton, ${ }^{2}$ Faye, ${ }^{3}$ Luc, ${ }^{2}$ Lasfargues, ${ }^{4}$ Paris, ${ }^{5}$ Agius, ${ }^{5}$ Stocks. ${ }^{1}$ APHP, Paris Descartes University, Paris, France, ${ }^{2}$ ANSES, Paris, France; ${ }^{3}$ INSERMU964, Vandoeuvre-Lès-Nancy, France; ${ }^{4}$ Occupational Diseases Department, University Hospital, INSSERMU964, Vandoeuvre-Lès-Nancy, France; ${ }^{5}$ Centre for occupational and environmental health, University of Manchester, Manchester, United Kingdom

\subsection{6/oemed-2013-101717.405}

Objective Here we present data comparing trends in surveillance of occupational diseases in France and the UK for allergic contact dermatitis attributed to chromate in cement (CDCr) and contact urticaria attributed to latex (UL) within the context of changes in legislation or of national actions to reduce exposure to allergens at these two countries workplace.

Methods The time period for analysis was selected to coincide with the introduction of legislation or compensation in the UK and France (2005 for cement and 2004 for latex). Using the data of two nationwide occupational diseases reporting networks in UK and France (THOR and RNV3P respectively), we analysed the temporal trends of CDCr and of UL over the period 1998-2009. We calculated reporting odd ratio (ROR) with reference to period preceding the change of legislation for the causal agent (cement or latex) relative to time period after intervention, both for British and French data. The ROR compares the ratio of incident cases attributed to one specific allergen relative to all other causal agents, before and after the change in legislation.

Results During 2006-2009 relative to reference period, there was a significant decline of $\mathrm{CDCr}$ in UK construction sector $(\mathrm{ROR}=0.37 ; 95 \%$ IC: $0.18-0.76)$ and a decline of french CD to cement $(\mathrm{ROR}=0.66 ; 95 \%$ IC: $0.0 .35-1.22)$. Concerning $\mathrm{UL}$, there was a decrease in UK healthcare workers $(\mathrm{ROR}=$ 0.84; 95\% IC: 0.57 - 1.25) in 2003-2007 period compared to $1998-2002$ and a decrease in french cases $(\mathrm{ROR}=0.73 ; 95 \%$ IC: 0.43 - 1.24).

Conclusion Through two distinct networks in UK and France, significant decline of $\mathrm{CD}$ attributed to chromate in cement and latex in gloves were observed following actions to reduce occupational exposure. These features are consistent with strong effectiveness of European preventive actions in these two countries.

\section{Session: 35. Occupational respiratory health}

\section{JAPANESE INDIUM COHORT STUDY: FIVE-YEAR FOLLOW-UP}

${ }^{1} \mathrm{M}$ N Nakano, ${ }^{2} \mathrm{~A}$ Tanaka, ${ }^{2} \mathrm{M}$ Hirata, ${ }^{1} \mathrm{~N}$ Yoshioka, ${ }^{1} \mathrm{~K}$ Omae. ${ }^{1}$ School of Medicine, Keio University, Tokyo, Japan; ${ }^{2}$ Graduate School of Medical Sciences, Kyushu University, Fukuoka, Japan

\subsection{6/oemed-2013-101717.406}

Background Since the demand for manufacturing flat panel display expands, we are ongoing an indium cohort study and followed up 310 indium exposure workers for 5 years to assess the changes on the lung effects.

Methods We conducted a baseline epidemiologic survey in 14 indium related plants between 2003 and 2006. A 5-year followup study was conducted in 11 of these 14 initial plants between 2008 and 2011. Follow-up rate is $57.2 \%$ (310/542). Indium concentration in serum (In-S) was determined as an exposure parameter, and effects on the lungs were examined. Some potential confounders were also checked. All participates were classified into three categories, non-, current, and former exposure, according to occupational history of indium exposure.

Results During the follow-up, intensive efforts for improvement of working environments were made in all 11 factories. In currently and formerly exposed workers, mean In-S and geometric means of interstitial biomarkers, KL-6 and SP-D, were significantly lower at follow-up than in the baseline study. In currently and formerly exposed workers, prevalence decreased by approximately $30 \%$ for KL-6 abnormalities but did not significantly decrease at all for $\mathrm{FEV}_{1.0} / \mathrm{FVC}, \% \mathrm{FVC}$, or $\% \mathrm{FEV}_{1.0}$ abnormalities. In the formerly exposed group, chest high-resolution computed tomography (HRCT) showed high prevalence of interlobular septal thickening and emphysematous change (15.1\% and $26.7 \%$, respectively). Aggravation of interstitial change on HRCT was not affected by In-S level, whereas that of emphysematous change progressed in a dose-response manner, even after adjustment for smoking and age. Workers with $\geq \operatorname{In}-\mathrm{S} 20 \mu \mathrm{g} / \mathrm{L}$ had a greater risk of aggravation of emphysematous findings (AOR: 13.31; 95\% CI: 3.29-53.89). During the 5-year-followup, two non-exposed worker at the baseline were diagnosed as lung and stomach cancers, and one currently exposed worker was diagnosed as renal cancer.

Conclusion Emphysematous changes might cause more chronic and longer-term lung effects than interstitial pneumonia.

\section{CHRONIC-OBSTRUCTIVE PULMONARY DISEASE (COPD) CAUSED BY INHALATION OF INORGANIC DUST AT THE WORKPLACE - SYSTEMATIC REVIEW AND METAANALYSIS}

${ }^{1} \mathrm{M}$ S Brueske, ${ }^{1}$ Thiering, ${ }^{1}$ Heinrich, 'Wichmann, ${ }^{2}$ Huster, ${ }^{2}$ Nowak. ${ }^{1}$ Helmholtz Zentrum München, Neuherberg, Germany, ${ }^{2}$ Ludwig-Maximilians-Universität, München, Germany

\subsection{6/oemed-2013-101717.407}

Objective A systematic review of the scientific medical literature on COPD and the inhalative exposure to inorganic dust and a metaanalysis. 